
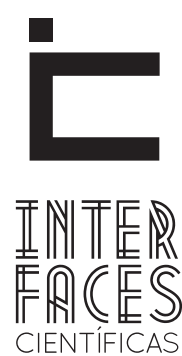

SAÚDE E AMBIENTE

\title{
PERFIL ESPERADO POR LABORATÓRIOS PARA CONTRATAÇÃO DE ANALISTAS CLÍNICOS EM ARACAJU-SE
}

Débora Machado Barreto ${ }^{1}$
Malone Santos Pinheiro²

\section{RESUMO}

O objetivo do presente estudo foi analisar o perfil esperado por laboratórios clínicos para contratação de analistas clínicos. A metodologia utilizada foi um questionário semiestruturado com perguntas referentes ao contratante e ao contratado. Nos resultados observou-se que o questionário foi respondido em $70 \%$ pelos Biomédicos e que estes tinham um bom tempo que trabalhavam na empresa, eles utilizavam o currículo, prova prática / teórica e a entrevista como método para contratação. Notou-se, também, que os contratantes, cuja maioria era responsável técnico e Biomédico, não priorizaram o sexo, $65 \%$ priorizavam o profissional graduado em
Biomedicina, com experiência profissional de um a dois anos e com pós-graduação lato sensu. Ressaltou-se que a qualidade dos Biomédicos está boa e que a média salarial está baixa quando se compara com outros autores. Observou-se que o perfil desejado pelos laboratórios clínicos pesquisados para contratação de analistas clínicos é de formação acadêmica em Biomedicina com dois anos de experiência profissional prévia.

\section{PALAVRA-CHAVE}

Analista clínico. Laboratório. Contratação. 


\section{ABSTRACT}

The purpose of this study was to analyze the profile expected by clinical laboratories for hiring clinical analysts. The methodology used was a semi-structured questionnaire with questions pertaining to the contractor and the engaged. The results found that the questionnaire was answered by $70 \%$ by the Biomedical and they had a good time working in the company, they used the curriculum, practical/theoretical test and interview as a method for hiring. We also note that contractors whose most were technically responsible and were biomedical not prioritized gender, $65 \%$ prioritized the professional degree in biomedicine, with experience of one to two years and lato sensus graduate. We emphasize that the quality of Biomedical is good and that the average salary is low when compared with other authors. We observed that the profile desired by clinical laboratories surveyed for hiring clinical analysts is of academic training in Biomedicine with two years of prior professional experience.

\section{KEYWORDS}

Clinical Analyst. Laboratory. Hiring.

\section{RESUMEN}

El objetivo de este estudio fue analizarla esperada el perfil esperado por laboratorios clínicos para la contratación de analistas clínicos. La metodología utilizada fue un cuestionario seme estructurado con preguntas referentes al contratante y al contratado. Los resultados que hemos observado, es que el cuestionario fue contestado por el $70 \%$ por la biomédica y éstos tenían un buen tiempo que trabajaban en la empresa. Ellos utilizaban el currículo, el plan de estudios, el examen práctico/ teórico y la entrevista como método para la contratación. También hay que destacar que los contratistas cuya mayoría era técnica responsables y Biomédicos, no priorizaron el género, $65 \%$ prioriza el título profesional en Biomedicina, con experiencia profesional de uno a dos años y de posgrado. Hacemos hincapié en que la calidad de la biomedicina es bueno y que el salario medio es bajo en comparación con otros autores. Hemos observado que el perfil deseado por los laboratorios clínicos es de formación académica en Biomedicina con dos años de experiencia profesional previa.

\section{PALABRAS CLAVE}

Analista clínico. De laboratorio. De empleo. 


\section{INTRODUÇ̃̃̃o}

O laboratório clínico vem aumentando sua significância devido à importância que os testes laboratoriais assumem na conduta clínica, onde até $70 \%$ das decisões clínicas no ambiente hospitalar como a conduta de internamento, alta e modificação no esquema terapêutico estão altamente associadas aos resultados dos exames laboratoriais (SANTOS; ANDRIOLO, 2008).

O desafio do laboratório é assegurar que os resultados produzidos reflitam, de forma fidedigna e consistente, a situação clínica apresentada pelos pacientes, garantindo que não haja interferências nesse processo. A informação produzida deve satisfazer as necessidades de seus clientes e possibilitar a determinação e a realização correta de diagnóstico, tratamento e prognóstico das doenças. O foco principal do laboratório é a melhoria contínua dos processos envolvidos no diagnóstico, é oferecer os melhores serviços para os clientes (CHAVES, 2010).

A melhoria contínua dos processos envolvidos deve representar o foco principal de qualquer laboratório clínico. Para isso, deve-se constantemente atentar para novas tecnologias, serviços, melhoria no atendimento aos clientes e possuir colaboradores treinados e atualizados (BONINI et al., 2002).

Estima-se que, estejam disponíveis aproximadamente 7.500 diferentes tipos de exames distribuídos nas diferentes áreas de atuação do laboratório clínico. A cada dia um número maior de indivíduos saudáveis tem sido submetido a baterias de testes com finalidades preventivas ou, mesmo, apenas administrativas que são os chamados exames de triagem (check-up) e os exames periódicos (SANTOS; ANDRIOLO, 2008). Devido à grande rotina, a implementação de processos automatizados em laboratórios clínicos nos últimos anos vem substituindo procedimentos manuais.
A automação no laboratório é utilizada com objetivo de diminuir o tempo na execução do exame, mantendo ou melhorando a qualidade e para reduzir o custo (AGUIAR \& PAZ, 2011; MIDDLETON, 2000), conceitua automação como a aplicação de técnicas computadorizadas ou mecânicas com o objetivo de tornar um processo mais eficiente, maximizando a produção com menor gasto de energia e gerando maior segurança.

O acompanhamento das inovações tecnológicas torna-se essencial para a sobrevivência do analista clínico no contexto de automação. Em um cenário onde elevado custos para implantação e manutenção de serviços praticamente impossibilitam iniciativas individuais. Os profissionais desse setor têm que estar atentos às modificações tecnológicas e mercadológicas para que simplesmente não sejam substituídos por equipamentos que fornecem resultados de forma mais ágil e precisa (SEKI et al., 2002).

No Brasil, três profissões estão habilitadas para atuar em análises clínicas: Biomédico, Farmacêutico Bioquímico e Médico Patologista (ANVISA, 2011). Esses profissionais possuem em seu currículo disciplinas como Parasitologia, Microbiologia, Bioquímica, Hematologia, dentre outras, que os habilitam a traduzir em linguagem clínica os resultados obtidos nos testes laboratoriais (SEKI et al., 2002; SOUZA et al., 2009). A escolha de profissionais competentes e competitivos torna-se de suma importância para a manutenção da qualidade laboratorial e pela busca de novos horizontes para o laboratório clínico (SOUZA et al., 2009).

Nesse cenário de constantes modificações no ambiente laboratorial, o perfil profissional dos analistas clínicos vem sofrendo alterações quanto às exigências mercadológicas, bem como, também é exigido das instituições de ensino superior modificações nas 
suas estruturas curriculares com intuito de preparar o profissional para o laboratório clínico moderno (AGUIAR, et al., 2011).

O objetivo desse estudo é investigar o perfil profissional do analista clínico esperado pelos laboratórios clínicos atuais, utilizando-se de formulários semiestruturados aplicados aos responsáveis pelas contratações nos laboratórios clínicos estudados.

\section{METODOLOGIA}

O material utilizado na pesquisa foi um questionário semiestruturado, que tem por finalidade buscar informações relevantes acerca das expectativas dos laboratórios clínicos, frente ao perfil profissional dos analistas clínicos. 0 referido questionário é composto por 5 questões subjetivas que se referiam ao contratante e 11 questionamentos objetivos referente ao candidato ou processo de contratação. O questionário foi direcionado para os laboratórios clínicos privados via e-mail para diversos estados do Brasil, acompanhado de informações importantes sobre a pesquisa com um apelo a colaboração voluntária e, também, por meio de visita nos laboratórios clínicos de Aracaju-SE para aplicação mais incisiva do questionário. A aplicação do questionário foi entre março e junho de 2011, totalizando 20 participantes na pesquisa.

\section{RESULTADOS}

Após o envio de 60 questionários, 20 foram respondidos plenamente, perfazendo um percentual de $33,3 \%$. Depois da compilação dos dados obtidos pôde-se determinar que a formação profissional dos respondentes, predominantemente foi de Biomédicos (70\%) seguidos por Farmacêuticos (15\%) e Administradores (10\%) e Médico Patologista Clínica (5\%). A pesquisa foi realizada em laboratórios de diversos portes que foram estratificados conforme tabela 1.
Tabela 1 - Informações referentes aos laboratórios estudados quanto à quantidade de funcionários

\begin{tabular}{lll}
\hline Quantidade de funcionários & $\mathbf{N}$ & \% \\
\hline$<5$ & 2 & 10 \\
$\mathbf{5}-10$ & 5 & 25 \\
$\mathbf{1 0 - 3 0}$ & 8 & 40 \\
$>\mathbf{3 0}$ & 5 & 25 \\
\hline
\end{tabular}

Fonte: Dados da pesquisa, 2011.

Quando questionados quanto ao cargo que ocupam na instituição contratante, os resultados demonstraram heterogeneidade conforme mostra na Figura 1. Em média estes profissionais tinham aproximadamente 9,5 anos de atuação na empresa, sendo 33 anos o indicador máximo e 2 meses o indicador mínimo e apresentaram, também, uma média de 12,9 anos de formados tendo máximo de 37 anos e mínimo de 1,6 anos.

Figura 1 - Valores percentuais referentes ao cargo ou função dos respondentes na empresa

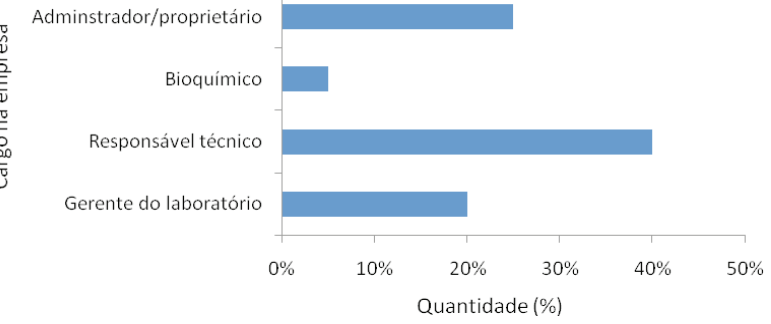

Fonte: Dados da pesquisa, 2011.

Para o questionamento acerca do poder de contratação, esse foi atribuído majoritariamente ao chefe do laboratório / responsável técnico / coordenador (45\%), seguido pelo dono da empresa (15\%) e a concomitância entre o responsável do laboratório e o departamento de recursos humanos (20\%). O processo de contratação se dá principalmente por análise curricular, entrevista e realização de prova prática ou teórica (40\%), seguida pela seleção por currículo e entrevista (20\%), e indicação (5\%). As análises dos resultados demonstraram que (65\%) dos labora- 
tórios priorizam a contratação de Biomédicos, $(20 \%)$ de Farmacêuticos e (35\%) deles demonstraram não terem prioridade por algum tipo de formação acadêmica. Quando questionados sobre o perfil do candidato a escolha por algum gênero em específico não foi priorizada por (65\%) dos respondentes. Porém, para os demais o gênero feminino foi citado por aproximadamente (57\%). Para os questionamentos referentes ao tempo de formação ideal do candidato e a experiência profissional prévia (65\%) dos contratantes demonstraram 1 a 2 anos de atuação.

No que concerne à qualidade de formação dos pretendentes à vaga de analista clínico, os contratantes acreditaram que (55\%) destes possuem uma formação acadêmica boa, enquanto que (30\%) classificaram como regular, $(10 \%)$ ruim e $(5 \%)$ péssimo, sendo que nenhum respondente acredita que essa formação é muito boa. Quanto ao questionamento sobre a influência de pós-graduação na contratação, (65\%) das respostas sinalizou preferir profissionais com títulos de pós-graduação, principalmente em especialização em lato sensu. Contudo $80 \%$ dos laboratórios demonstraram não haver diferenciação salarial entre especialistas e graduados.

Quando questionados acerca da preferência profissional entre técnicos de nível médio e superior para a realização dos exames laboratoriais, as respostas denotaram uma prioridade para o nível superior conforme Figura 2.

Figura 2 - Valores percentuais referentes à preferência de profissionais para a realização de exames laboratoriais

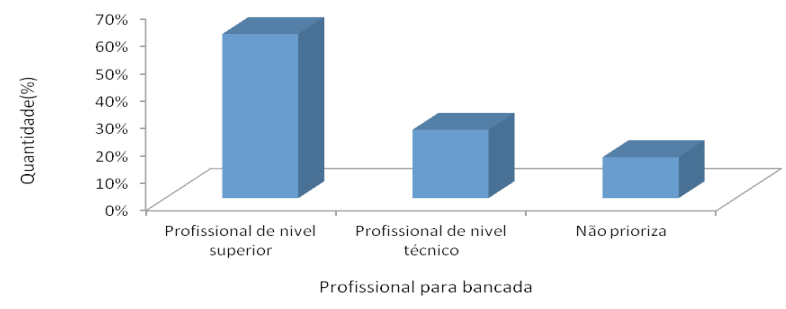

Fonte: Dados da pesquisa, 2011
Para o questionamento referente à faixa de remuneração dos profissionais analistas clínicos, os resultados demonstraram certa heterogeneidade de informação conforme Figura 3.

Figura 3 - Valores percentuais referentes à remuneração mensal dos analistas clínicos

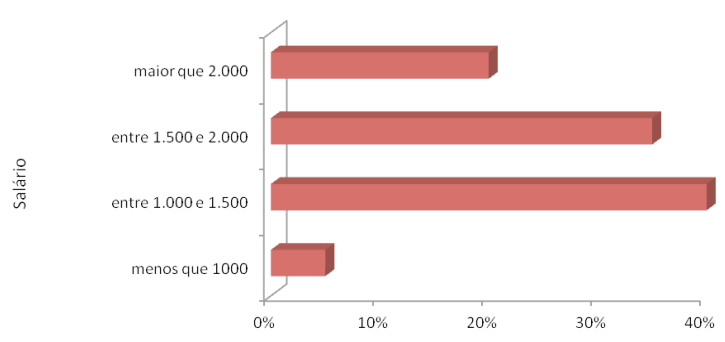

Fonte: Dados da pesquisa, 2014.

\section{DISCUSSÃO}

Do total de questionários enviados, apenas (33,3\%) retornaram. Uma pesquisa foi enviada questionários pelo correio $(39,5 \%)$ retornaram. Já outra pesquisa enviou pela internet, onde $(25,3 \%)$ retornaram. Dessa forma, pesquisas não presencial talvez não favoreçam grande adesão que pode estar atrelado à insegurança de endereços eletrônicos desconhecidos ou pela falta de hábito de olhar e-mail frequentemente.

O perfil do profissional contratante nesse estudo foi caracterizado por uma formação técnica (Biomedicina / Farmácia), com experiência média de 13 anos de exercício profissional e 10 anos de atuação na empresa.

Para contratação foi observado que a principal metodologia relacionada ao processo seletivo foi análise curricular, entrevista e prova teórica / prática. Tal fato está relacionado à possibilidade de conter as experiências profissionais no currículo, observar características comportamentais, como autocontrole e segurança na entrevista e o nível de conhecimento técnico por meio de provas teóricas / práticas. 
Para 65\% dos laboratórios clínicos o profissional Biomédico tem preferências no momento da contratação, frente aos outros profissionais habilitados para atuarem nesse segmento. Esse fato pode estar relacionado à tradição do curso de Biomedicina em Sergipe de que vem formando profissionais desde 1998, sendo o mais antigo formador de analistas clínicos em Sergipe. Outros fatos podem estar relacionados a essa preferência como a ausência de um acordo trabalhista da classe, estipulando um piso salarial e carga horária e um currículo que melhor prepara o profissional para exercício da profissão contendo disciplinas aplicadas a diversas áreas e um elevado número de horas destinadas a atividades práticas.

$\mathrm{Na}$ presente pesquisa foi demonstrado que o analista clínico possui faixa salarial entre R\$1.000 e R\$1.500, o que comparado outros estudos similares demonstra que Sergipe aplica um piso salarial mais baixo que a média nacional.

Em uma pesquisa realizada em 2009, foram estudados diversos cursos de graduação, onde não houve diferença estatisticamente significativa entre o gênero de ingressantes e concluintes, indicando uma estabilidade do aumento da participação feminina nos cursos da área de saúde, fato que coincide com a opinião dos contratantes nesse trabalho, que não demonstraram preferência quanto ao gênero dos analistas clínicos no ato da contratação.

\section{CONCLUSÃO}

Apesar da dificuldade de adesão dos participantes à pesquisa, pôde-se observar que o perfil desejado pelos laboratórios clínicos pesquisados para contratação de analistas clínicos é de formação acadêmica em Biomedicina com dois anos de experiência profissional prévia, sem preferência por gênero e com pós- graduação lato sensu, apesar dessa não influenciar no aumento da remuneração desse profissional. Quanto ao poder de contratação esse foi atribuído, em sua maioria, aos responsáveis técnicos pelos laboratórios estudados, os quais possuíam predominantemente formação acadêmica em Biomedicina e experiência de aproximadamente 10 anos de atuação na empresa. Foi observado ainda que a metodologia de seleção dos candidatos mais aplicada foi a análise curricular, entrevista e realização de prova prática / teórica.

\section{REFERÊNCIAS}

AGUIAR, G. C; PAZ, C. O. Conceitos de automação na medicina laboratorial: revisão de literatura. J Bras Patol, v. 47, n. 2, p. 119-127, 2011.

ANVISA. Disponível em: <http://www.anvisa.org.br>. Acesso em: 30 maio 2011.

BASTOS, J. R. M et al. Análise do perfil profissional de cirurgiões-dentistas graduados na faculdade de odontologia de Bauru - USP entre os anos de 1996 e 2000. J Appl Oral Sci, v.11, n.4, 2003, p. 283-289.

BONINI, P. et al. Errors in Laboratory Medicine. Clinical chemistry, v.48, 2002, p. 691-698.

CHAVES, C. D. Controle de qualidade no laboratório de análises clínicas. J Bras Patol, v. 46, n.5, 2010.

MIDDLETON. S. R. Developing an Automation Concept That Is Right for Your Laboratory. Clinial Chemistry, v. 46, 2000, p. 757-763.

PIMENTA, J. R. F.; FERNANDES, N. S. A importância da coletivização do processo trabalhista. Revista do Tribunal Regional do Trabalho da 3a. Região, v.46, n.76, 2007, p. 45-60.

RODRIGUES, K. M.; PERES F.; WAISSMANN, W. Condições de trabalho e perfil profissional dos nutricionistas egressos da Universidade Federal de Ouro Pre- 
to, Minas Gerais, entre 1994 e 2001. Ciências e saúde coletiva. v.12, n.4, 2007.

SANTOS, C. E. F.; ANDRIOLO, A. Intervalos de referência no laboratório clínico. Jornal Brasileiro de Patologia e Medicina Laboratorial. v.44, n.1, Rio de Janeiro, 2008.

SAÚDE WEB. Disponível em: <http://saudeweb.com. br>. Acesso em: 8 jun. 2011.

SEKI, M.; OKABI, M. S; GOMES, P. P. J. Contribuição para discussão segmentada sobre mercado de traba- lho e perspectivas profissionais em patologia clínica/ medicina laboratorial. J Bras Patol, v. 38, n. 3, 2002, p. 205-206.

SEKI, M. et al. A inovação de valores nos laboratórios clínicos. J Bras Patol, v. 39, n. 3, 2003, p. 211-214.

SOUZA, P. S. et al. Laboratórios clínicos: evidências e vislumbres para credenciamento frente às conveniadoras particulares. Newslab. n.93, 2009. 